\title{
Preparation of controlled release microspheres using supercritical fluid technology for delivery of anti-inflammatory drugs
}

\author{
Ana Rita C. Duarte ${ }^{\mathrm{a}, *}$, Mariana Sousa Costa ${ }^{\mathrm{b}}$, Ana Luísa Simplício ${ }^{\mathrm{a}}$, \\ Maria Margarida Cardoso ${ }^{b}$, Catarina M.M. Duarte ${ }^{\mathrm{a}}$

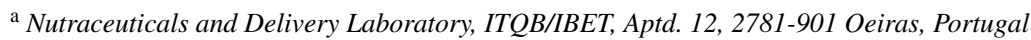 \\ ${ }^{\mathrm{b}}$ REQUIMTE/CQFB, Departamento de Química, FCT-UNL, 2829, 516 Caparica, Portugal
}

Received 16 August 2005; received in revised form 7 November 2005; accepted 8 November 2005

Available online 20 December 2005

\begin{abstract}
Ethylcellulose/methylcellulose blends were produced using different precipitation techniques and impregnated with naproxen, a non-steroidal anti-inflammatory drug (NSAID). Solvent-evaporation technique was used not only for the preparation of ethylcellulose/methylcellulose microspheres but also to encapsulate naproxen. Supercritical fluid (SCF) impregnation was also performed to prepare naproxen loaded microspheres. The microspheres, impregnated by the SCF technique, were prepared both by solvent-evaporation and by a supercritical antisolvent (SAS) process. In vitro release profiles at $\mathrm{pH} 7.4$ and 1.2, of naproxen-loaded microspheres were evaluated and the results were modelled Fick's law of diffusion and Power law. Miscrospheres prepared by supercritical antisolvent have a higher loading capacity and present a slower release profile. The systems studied present a release mechanism controlled by drug diffusion which complies Fick's law of diffusion.
\end{abstract}

(C) 2005 Elsevier B.V. All rights reserved.

Keywords: Controlled delivery systems; Supercritical fluids; SAS; Ethylcellulose; Anti-inflammatory drugs

\section{Introduction}

Controlled drug delivery products, using biocompatible or biodegradable polymers, have received considerable attention in the last years. These substances provide in general a more controlled rate of assumption of the drug by the body improving its therapeutic action. In fact, there is a growing interest of the pharmaceutical industry in the development of these systems (Heller, 1996).

In many cases, conventional drug delivery products provide sharp increases in the drug concentration at potentially toxic levels, followed by a relatively short period at the therapeutic level and drug concentration drops until new administration (Freiberg and Zhu, 2004). Controlled drug delivery occurs when a polymer/drug system is designed to release the drug in a predetermined manner. The main purpose of these controlled release systems is to achieve a more effective therapy, i.e., a system with a delivery profile that would yield a high blood level of the drug

\footnotetext{
* Corresponding author.

E-mail address: rduarte@itqb.unl.pt (A.R.C. Duarte).
}

over a long period of time, avoiding the large fluctuations in drug concentration and to reduce the need of several administrations (Lisa Brannon-Peppas, 1997).

The preparation of drug release products necessitates the use of a mobile phase to dissolve and carry the drug component, which also swells and stretches the polymer matrix, facilitating the diffusion of the drug, and increasing the rate of impregnation. Conventionally, the preparation of these systems involves three steps: solubilization of the pharmaceutical in an appropriate solvent, diffusion of the pharmaceutical through the polymer and elimination of the residual solvent.

Impregnation using supercritical fluid technology has proven to be feasible when the pharmaceutical compound is soluble in carbon dioxide and the polymer can be swollen by the supercritical fluid. A high purity product, free of residual solvents is obtained, since no organic solvents are involved in the impregnation process (Kikic and Vecchione, 2003; Elvira et al., 2004). Supercritical fluids, especially supercritical carbon dioxide $\left(\mathrm{scCO}_{2}\right)$ have been identified as prime candidates to develop alternative clean processes for the preparation of drug-loaded polymeric matrixes (Kikic and Sist, 2000). Furthermore, the use of supercritical fluids can take advantage of their high diffu- 
sivity in polymers, in combination with the high solubility and plasticizing action. Therefore, compressed $\mathrm{CO}_{2}$ is considered a good drug carrier into biocompatible polymers. Moreover, when depressurisation occurs, the gas rapidly diffuses out of the polymer, deplasticizing it and warranting the complete removal of solvent, without exposing polymers and drugs to high temperatures, which may degrade them (Berens et al., 1992; Kazarian, 2004).

Polymer nanosuspensions of non-steroidal anti-inflammatory (NSAIDs) agents have been proposed as controlled delivery systems able to solve pharmacokinetic problems and some typical side effects of most of these drugs, namely, gastrointestinal adverse side-effects. The preparation of controlled release systems for these pharmaceutical compounds is the leading way to overcome this problem.

Although many polymers are used in the pharmaceutical industry, cellulose derivatives are among the most commonly used (Rekhi and Jambhekar, 1995). Ethylcellulose is a hydrophobic material used in a variety of applications such as sustained release and taste masking. It has been widely used to prepare controlled delivery systems of water-soluble materials. The drug release can be controlled to some extend by addition of a water-soluble or water swellable polymer, as methylcellulose (Handbook of Pharmaceutical Excipients, in press).

Being the most part of NSAIDs lipophilic drugs, the solvent evaporation method is generally used for their microencapsulation. However, during the microencapsulation process, a partial crystallization of the free drug can take place, which is undesirable since its release is not going to be controlled by the polymeric matrix (Leo et al., 2000).

In this work, in vitro release profiles of naproxen-loaded microspheres prepared by different techniques were investigated. Solvent-evaporation technique was used to produce not only ethylcellulose/methylcellulose microspheres but also to encapsulate naproxen. Supercritical fluid impregnation was performed to prepare controlled release naproxen microspheres. The microspheres were prepared both by solvent-evaporation and by supercritical antisolvent process (SAS). The SAS process is the supercritical precipitation process most commonly used, since non-soluble solutes in carbon dioxide can be processed. This is also the technique focused in this work. The principle of this process is to decrease the solvent power of the liquid by addition of an antisolvent $\left(\mathrm{CO}_{2}\right)$ in which the solute is insoluble (Jung and Perrut, 2001; Reverchon et al., 2001).

\section{Experimental procedure}

\subsection{Materials}

Ethylcellulose (48.0-49.5\% w/w ethoxyl content) (CAS 9004-57-3) Methylcellulose, with an intrinsic viscosity $3160 \mathrm{mPa}$ (CAS 9004-67-5) were purchased from Fluka. Naproxen (CAS 22204-53-1, 98.0\% purity) was purchased from Sigma. Dichloromethane, DCM (CAS 78-09-2, 99.9\% purity), Dimethylsulphoxide, DMSO (CAS 67-68-5, 99.9\% purity) and Acetone (CAS 67-64-1, 99.5\% purity) were purchased from Vaz
Pereira. Carbon dioxide (99.998 mol\%) was supplied by Air Liquide. All chemicals were used with no further purification.

\subsubsection{Preparation of microspheres}

2.1.1.1. Solvent-evaporation technique. Drug-free and microparticles containing naproxen were prepared by the solvent evaporation method. $2 \mathrm{~g}$ of ethylcellulose were dissolved in $20 \mathrm{ml}$ of the organic solvent (dichloromethane, in some cases containing 5 or $10 \mathrm{ml}$ of acetone). When preparing naproxenloaded microparticles, $1.2 \mathrm{~g}$ of drug was added to this solution. The solution of organic phase was slowly poured into $250 \mathrm{ml}$ of a $2.5 \% \mathrm{w} / \mathrm{v}$ (in some cases $5 \% \mathrm{w} / \mathrm{v}$ or $7.5 \% \mathrm{w} / \mathrm{v}$ ) methylcellulose aqueous solution, which acts as an emulsifying agent, and stirred with a two-baffled rotator for $1 \mathrm{~h}$ at a controlled stirring speed of $800 \mathrm{rpm}$. The formed oil-in-water (o/w) emulsion was then gently stirred at room temperature until complete evaporation of the solvent. Microparticles were then collected by centrifugation at $10000 \mathrm{rpm}$ for $20 \mathrm{~min}$ (B. Braun, model Sigma 4K10), washed 4 times with distilled water, filtered (Millipore, pore size $0.45 \mu \mathrm{m}$ ) and dried in a desiccator under vacuum at room temperature.

2.1.1.2. Supercritical antisolvent (SAS) technique. A solution of ethylcellulose + methylcellulose was prepared in $\mathrm{DCM}+\mathrm{DMSO}$ to be injected in a SAS apparatus. In order to change the porosity of the polymeric matrix a water-soluble solvent, acetone, was added to the solution. The apparatus works in a continuous co-current mode and it consists in a precipitator in which the antisolvent and the liquid solution are separately fed to the top of the chamber and are continuously discharged from the bottom (Fig. 1). The liquid solution is delivered into the precipitation chamber through a stainless steel nozzle $(100 \mu \mathrm{m})$. A filter of sintered steel with $0.1 \mu \mathrm{m}$ porosity is placed at the bottom of the vessel to collect the particles produced. The solvents are separated and recovered from a second vessel. Usually the temperature of the vessel is around $15^{\circ} \mathrm{C}$ and pressure 5-10 bar. The experiments were carried out all at the same operational conditions ( $80 \mathrm{bar}, 35^{\circ} \mathrm{C}, \mathrm{CO}_{2}$ flow $8.5 \mathrm{~L} / \mathrm{min}, 1 \mathrm{~mL} / \mathrm{min}$ ).

\subsubsection{Supercritical impregnation process}

The impregnation is performed in a semi-continuous apparatus described schematically in Fig. 2.

A tubular batch extraction cell is initially loaded with the pharmaceutical compound and packed between a sequence of a filter paper, cotton and net metallic discs that work as filters of small particles. The polymer is loaded, in the same manner, in the impregnation cell. The cells are then immersed in a thermostatized water bath heated by means of a controller that maintains temperature within $\pm 0.1^{\circ} \mathrm{C}$ (TC) (Ero Electronic LMS-491-13). Carbon dioxide is pumped into the cells using high pressure piston pump (Haskel model MCPV-71) until the operational pressure is attained. In the intervening time the cells are not connected to each other, however they are at the exact same pressure. The pressure inside the cells is measured with a pressure transducer (P) (LEO2 0.300 bar). The solubilization of the drug in the supercritical fluid and the swelling of the polymer due to the presence of carbon dioxide take place during one hour, a typical equilibrium time. Preliminary experiments with other 


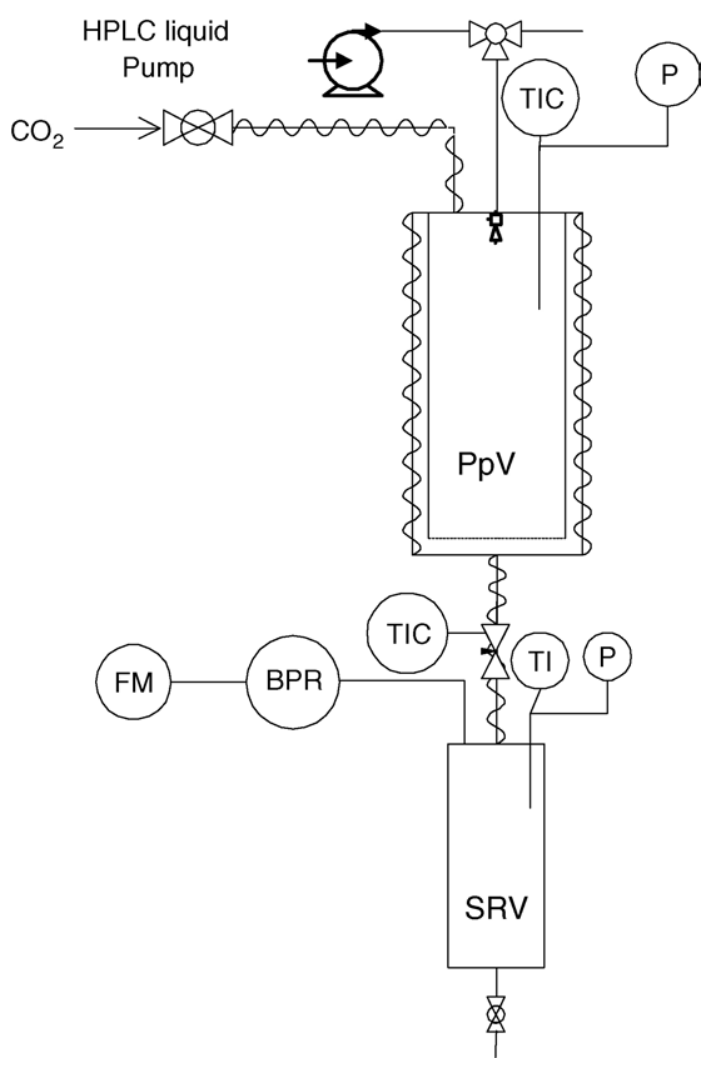

Fig. 1. SAS apparatus (TIC, temperature transducer; TI, temperature indicator; $\mathrm{P}$, pressure transducer; BPR, back pressure regulator; FM, flow meter; PpV, precipitation vessel; SRV, solvent recuperation vessel).

contact times were performed and similarly to other polymers and common model drugs, $1 \mathrm{~h}$ was found to be the adequate contact time for both the swelling of the polymeric matrix and solubilisation of the drug in $\mathrm{scCO}_{2}$. Opening valve V1 the cells are placed in contact and the continuous process of impregnation starts. A saturated stream of the pharmaceutical compound in carbon dioxide passes through the polymeric matrix, for a predetermined period of time and at a very slow rate so that the impregnation can occur. The outflow is regulated by a metering valve (Hoke 1315G4Y), in order to maintain a constant pressure in the system and a slow carbon dioxide flow, which is measured with a flowmeter (FM) (Alexander Wright DM3C). The solid that might be solubilized in the gas is collected in a small glass trap (T). The impregnation time for all the experiments

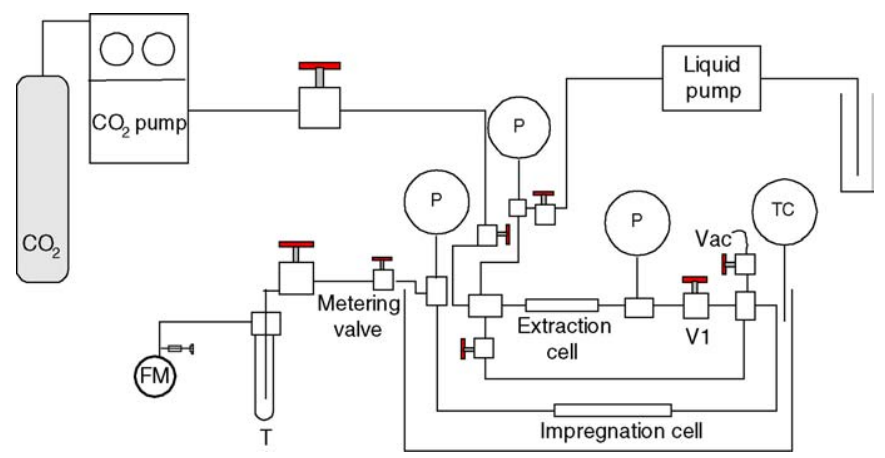

Fig. 2. Schematic representation of the impregnation apparatus. presented was $2 \mathrm{~h} 30 \mathrm{~min}$. At the end of this period the system is quickly depressurized and the impregnated sample is collected from the impregnation cell.

\subsubsection{Microspheres characterization}

2.1.3.1. Scanning electron microscopy (SEM). Samples of the precipitated powder were observed by a ZAISS 960 scanning electron microscope (SEM). The particles were fixed by mutual conductive adhesive tape on aluminium stubs and covered with gold palladium using a sputter coater.

2.1.3.2. In vitro drug release studies. Drug impregnated microspheres $(20 \mathrm{mg})$ were suspended in $40 \mathrm{ml}$ of phosphate buffer solution ( $\mathrm{pH}$ 7.4) and simulated gastric fluid without pepsin (pH 1.2) stirred at $150 \mathrm{rpm}$ at $37^{\circ} \mathrm{C}$. A $0.5 \mathrm{ml}$ aliquots were withdrawn at predetermined time intervals and the same volume of fresh medium was added to the suspension. The samples were filtered $(0.45 \mu \mathrm{m}$, Millipore filters $)$ and analysed by HPLC (ThermoFinnigan Surveyor consisting of a quaternary pump, autosampler and diode array detector). A C18 column (Merck Lichrospher $250-4,5 \mu \mathrm{m}$ ) kept at $30^{\circ} \mathrm{C}$, was used in isocratic conditions with a mobile phase consisting of water:acetonitrile:acetic acid (500:490:10). The flow rate was $1.2 \mathrm{ml} / \mathrm{min}$ and the volume of injection was $20 \mu \mathrm{l}$. Quantitation was performed at $280 \mathrm{~nm}$ by external standard calibration.

The total mass of released drug in each moment of the experiment was calculated taking into account the aliquots taken and the dilution produced by addition of fresh buffer.

\section{Mathematical modelling of drug release}

The knowledge of the mass transport mechanisms and the kinetics of the drug release are essential for the design of new drug delivery systems. Several models from simple empirical or semi-empirical to more complex mechanistic theories have been proposed to describe drug release from delivery systems (Higuchi, 1961; Siempmann and Peppas, 2001).

A proportionality between the fractional amount of drug released and the square root of time can as well be described by an exact solution of Fick's law of diffusion (Crank, 1975, 1968):

$\frac{M_{\mathrm{t}}}{M_{\infty}}=4\left(\frac{D_{\mathrm{t}}}{\pi \ell^{2}}\right)^{1 / 2}=k^{\prime} \sqrt{t}$

where $l$ is the thickness of the system and $k^{\prime}$ is the kinetic constant. The linearity of this equation can only be verified up to $60 \%$ of the total amount of drug $\left(M_{\infty}\right)$.

Another case is one where the drug release rate is independent of time, i.e., it corresponds to a zero-order kinetics. Many situations of release processes fall between these cases, the Fickian diffusion and the zero-order kinetics. An heuristic equation, known as power law, that translates this behaviour can be written as (Ritger and Peppas, 1987a, 1987b):

$$
\frac{M_{\mathrm{t}}}{M_{\infty}}=k t^{n},
$$


where $k$ is a constant and $n$ is the diffusional exponent, which is indicative of the transport mechanism. Like Eq. (1) this relationship is only valid for the first $60 \%$ of the drug released.

\section{Results}

Microspheres of ethylcellulose/methylcellulose were prepared by solvent-evaporation technique and using a supercritical antisolvent process. Particles produced using supercritical technology present a small diameter and a narrower size distribution (Fig. 3).

Comparing the particle size distribution of the pure ethylcellulose with one of the blends micronized by SAS there is a greater polydispersity, which is due to the fact that in the later case we two polymers are being co-precipitated. Nevertheless, the supercritical antisolvent process still presents enormous advantages when comparing with conventional techniques such as solvent evaporation. Table 1 presents the characteristics of the particles produced.

The release profiles of the different systems prepared were performed. For the same polymeric blend is clear that the techniques used to both prepare and impregnate the microspheres influence the percentage of impregnated drug. Fig. 4 illustrates the release profile in terms of drug concentration. The trend of the curves are different suggesting that they present different kinetics, therefore different release rates.

The release behaviour presented is similar for each blend impregnated. The process technique used to prepare the delivery system is clearly a factor that influences the release rates. The release rates are higher when the microspheres were prepared using the solvent-evaporation technique and lower in the case that particles were prepared by the conventional technique and impregnated with $\mathrm{CO}_{2}$. Particles prepared by solvent-evaporation and impregnated using supercritical fluid technology present a very slow release profile, only $40 \%$ of
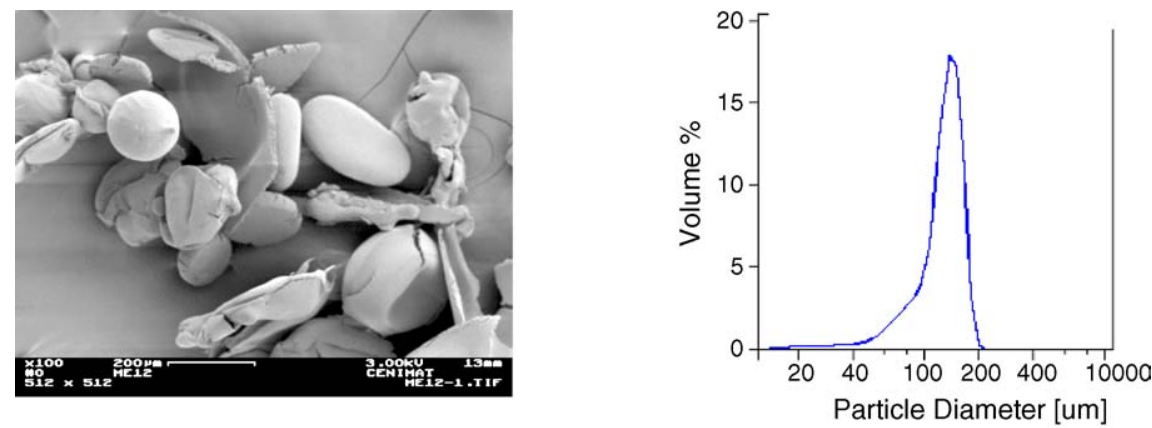

Ethylcellulose/Methylcellulose $(0.25 \% \mathrm{w} / \mathrm{v})$ solvent-evaporation
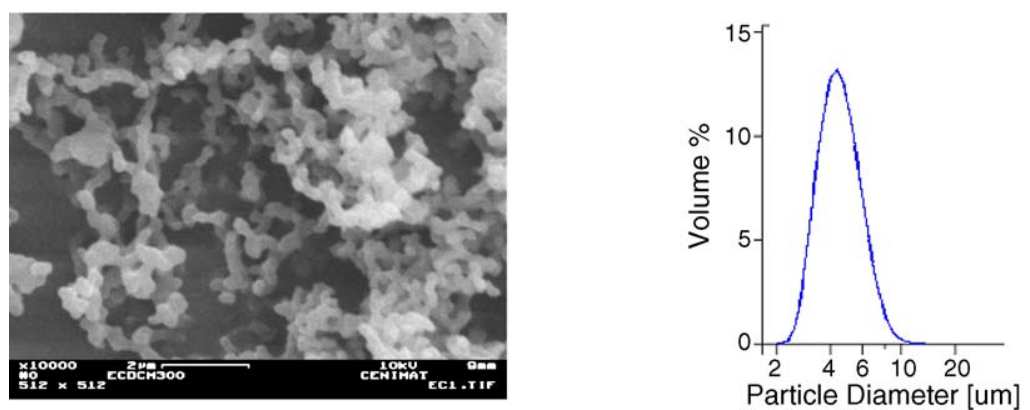

Ethylcellulose micronized by SAS
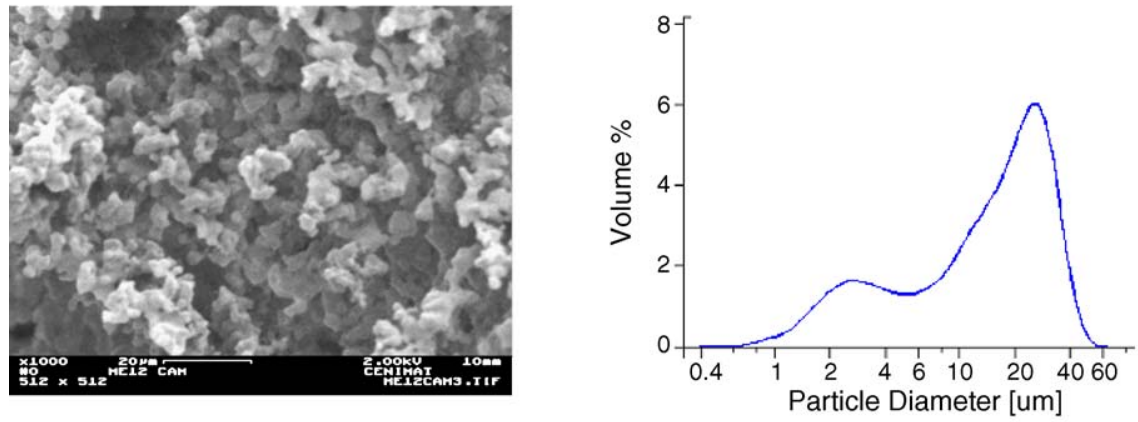

Ethylcellulose/Methylcellulose $(0.25 \% \mathrm{w} / \mathrm{v})$ micronized by SAS

Fig. 3. SEM images and particle size distribution of the particles prepared. 
Table 1

Characteristics of the particles prepared

\begin{tabular}{|c|c|c|c|c|c|c|}
\hline & System & Method of preparation & Method of impregnation & $\operatorname{MSD}(\mu \mathrm{m})$ & w/v \% methylcellulose & $\%$ impregnation $^{\mathrm{b}}$ \\
\hline 1 & Ethyl/methyl & Solv-evap & Solv-evap & 126.7 & 0.25 & 57.7 \\
\hline 2 & Ethyl/methyl & Solv-evap & Solv-evap & 225.7 & 0.5 & 63.3 \\
\hline 3 & Ethyl/methyl & Solv-evap & Solv-evap & 149.5 & 0.75 & 76.3 \\
\hline 5 & Ethyl/methyl & Solv-evap & Solv-evap & 130.6 & $0.25^{\mathrm{a}}$ & 65.2 \\
\hline 6 & Ethyl/methyl & Solv-evap & SCF impregnation & 126.7 & 0.25 & 1 \\
\hline 10 & Ethyl/methyl & Solv-evap & SCF impregnation & 130.6 & $0.25^{\mathrm{a}}$ & 0.9 \\
\hline 11 & Ethyl/methyl & SAS & SCF impregnation & 16.91 & 0.25 & 4.1 \\
\hline 12 & Ethyl/methyl & SAS & SCF impregnation & 17.65 & 0.5 & 3.2 \\
\hline 13 & Ethyl/methyl & SAS & SCF impregnation & 23.12 & $0.25^{\mathrm{a}}$ & 2.1 \\
\hline 14 & Ethylcellulose & SAS & SCF impregnation & 4.71 & - & 3.2 \\
\hline
\end{tabular}

${ }^{\text {a }}$ Particles prepared in the presence of acetone $(4,9,13$ with $25 \%$ and 5,10 with $50 \%)$.

${ }^{b}$ Relative quantity of drug in an impregnated sample, expressed in w/w percentage.
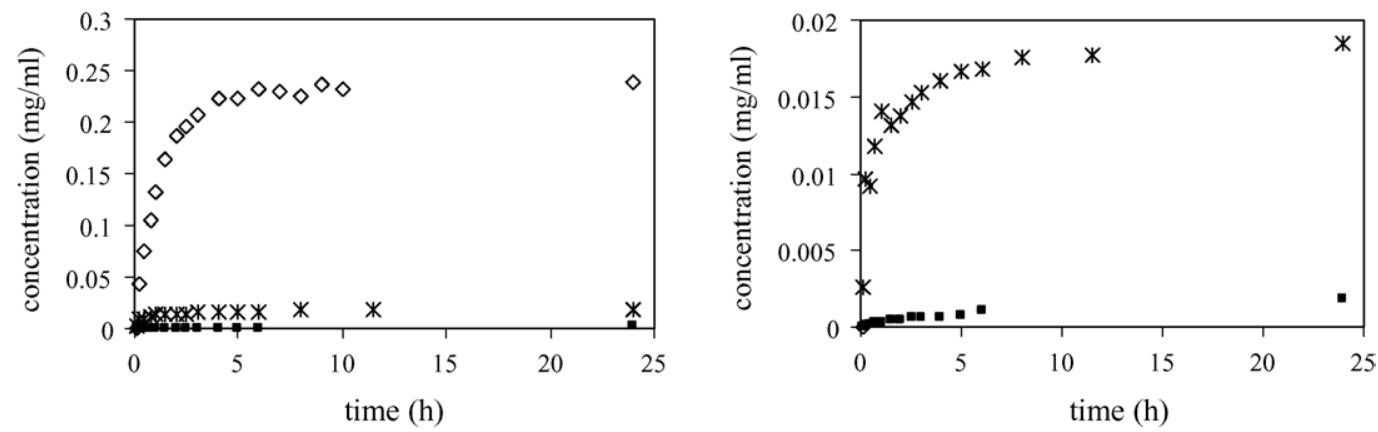

Fig. 4. Release profiles (pH 7.4) of the systems prepared with $0.25 \%(w / v)$ methylcellulose by solv-evap.

the drug is released after $8-10 \mathrm{~h}$. Considering that these microspheres were prepared for oral medication and since the gastrointestinal transit time is considered to be $6-8 \mathrm{~h}$, as reported by Davis (1985), the particles prepared using solvent evaporation or supercritical fluid technology seem to be the most promising ones. Nonetheless, the conventional method for the preparation of the microspheres presents the drawbacks already pointed out. So, supercritical fluid technology presented very promising results in the preparation of new drug delivery systems.

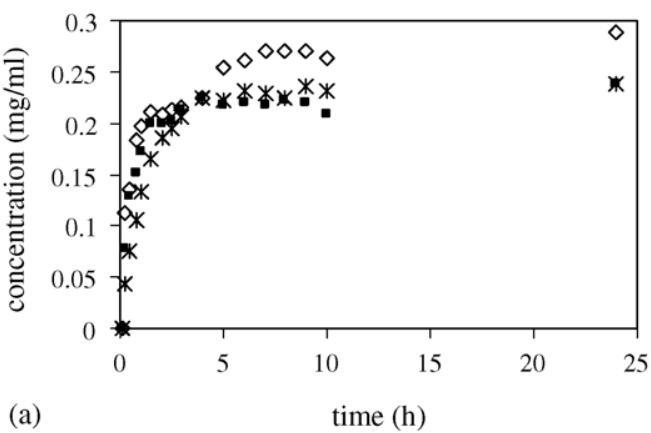

Regarding the different formulations, interesting results were obtained. The formulation process has a much greater impact on the release profile when supercritical fluids are used in the impregnation step. Fig. 5(a) and (b) represent respectively the release profiles of the different formulations prepared with the conventional technique of solvent-evaporation and when microspheres prepared conventionally were impregnated using $\mathrm{CO}_{2}$. When naproxen-loaded particles were prepared by solventevaporation the release rates are similar as the initial slope of the release profile curve is similar. However, when the particles were

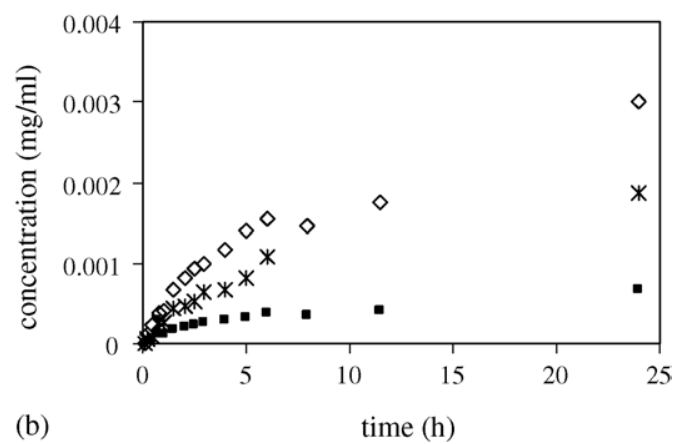

Fig. 5. Release profiles ( $\mathrm{pH} 7.4$ ) of the systems prepared by (a) solvent-evaporation technique and (b) solv-evap + sc impregnation with (*) $0.25 \%$ (w/v), ( $\mathbf{\square}) 0.5 \%$ $(\mathrm{w} / \mathrm{v}),(\diamond) 0.75(\% \mathrm{w} / \mathrm{t})$ methylcellulose. 

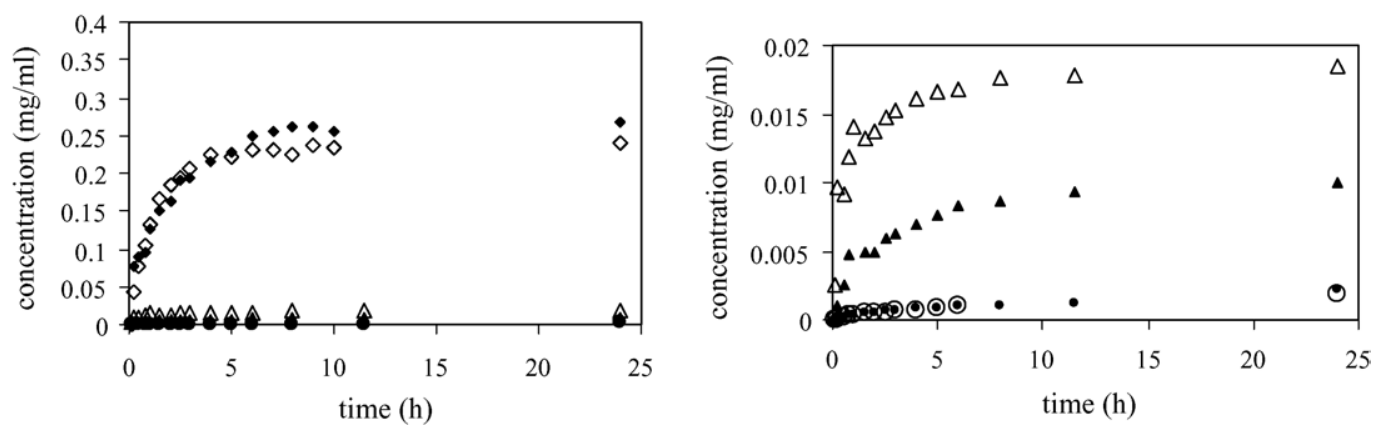

Fig. 6. Release profiles (pH,7.4) of the systems prepared in the presence of $0 \%$ (open symbols) and $25 \%$ acetone (filled symbols): $(\diamond)$ and $(\diamond)$ solvent-evaporation technique; $(\bigcirc)$ and $(\bullet)$ solv-evap + sc impregnation; $(\Delta)$ and $(\boldsymbol{\Lambda})$ SAS + sc impregnation with $0.25 \%(\mathrm{w} / \mathrm{v})$ methylcellulose.

prepared by solvent evaporation and then impregnated using supercritical fluid technology the trends of the curves are different leading to different initial slopes, and therefore different release kinetics.

The presence of acetone in the preparation of the microspheres does not affect the kinetics of the release and the drug release rate is the same as the initial slopes are also the same (Fig. 6). The release profiles of the samples were also evaluated at two different pHs 1.2 and 7.4, simulating respectively the gastric and intestinal environment (Fig. 7). All the formulations prepared and impregnated by solvent evaporation and the ones prepared by SAS and impregnated using supercritical fluids present an almost insignificant release rate in acidic solution, meaning that the release would mainly occur in the intestine. This is due to the fact that there is a higher drug release rate in these systems. When particles were produced by solvent evaporation and then were impregnated using supercritical fluids the difference is not significant since only a low percentage of drug is released.

To better understand the drug release mechanism of the system prepared, Fick's law of diffusion and Power law have been applied. Fick's law fitted well the data up to $60 \%$ of release for all experiments.

The power law is another simple semi-empirical equation that was applied to the results obtained. Although there is a very good correlation between the percentage of drug released and the time of release, the release exponent obtained did not always fit the values established for the case of a spherical sample (Siempmann and Peppas, 2001). Peppas et al. have studied the effect of the particle size distribution on the calculated diffusional exponent and have come to the conclusion that the "limits" set for spherical samples can only be applied if the particles are monodisperse

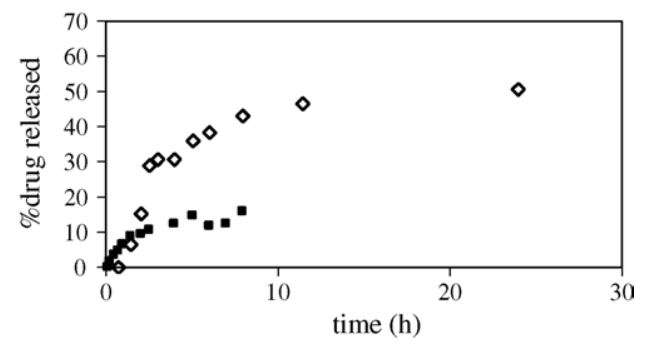

Fig. 7. Release profiles of the system prepared using the SAS + sc fluid impregnation $(0.25 \%$ (w/v) methylcellulose (25\% acetone)): $(\diamond) \mathrm{pH} 7.4 ;(\square) \mathrm{pH} 1.2$.

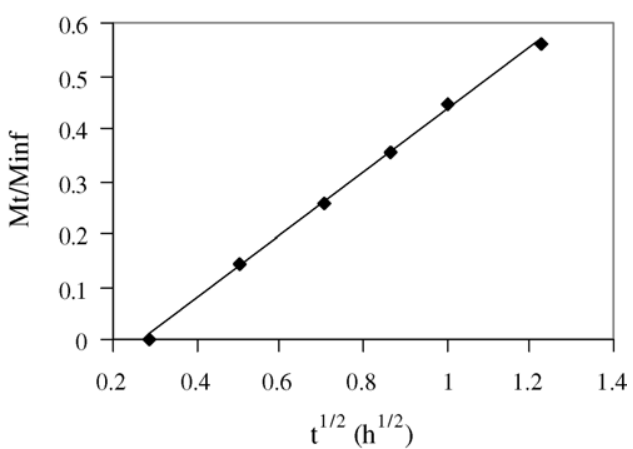

Fig. 8. Release profile of the system prepared by solvent-evaporation $(0.25 \%$ (w/v) methylcellulose): ( ) experimental data; (一) Fick's law of diffusion.

(Ritger and Peppas, 1987), that was the case when particles were prepared by SAS. In this case, the exponent values obtained when Power law was applied to experimental data, were found to be in the range of $0.43-0.85$, meaning that the release was an anomalous Fickian behaviour (Fig. 8).

In Table 2, the kinetic constants determined based on the Fickian model, for experiments 1, 6 and 11 are presented.

When the same technique was used to prepare and impregnate the particles, the kinetics constants were similar, namely particles prepared and impregnated by solvent evaporation and particles prepared by SAS and impregnated using supercritical fluid technology. These kinetic constants were higher than in the case when particles were prepared by solvent-evaporation and impregnated using supercritical fluid technology. In this case, the impregnated particles present a considerably large diameter. This, combined with the fact that the drug carried by the supercritical fluid, during the impregnation process, is entrapped in the core of the particle (due to the high diffusivity of carbon dioxide through the polymer matrix) and the longer diffusion path, leads to a slower release of the drug.

Table 2

Kinetic constant calculated from Fick's model, for the impregnation experiments with the system $0.25 \%$ w/v methylcellulose

\begin{tabular}{ll}
\hline Experiment & $k_{1}$ \\
\hline 1 & 0.6 \\
6 & 0.1 \\
11 & 0.5 \\
\hline
\end{tabular}




\section{Conclusions}

Supercritical fluid technology for the preparation of naproxen-loaded ethylcellulose/methylcellulose microspheres for controlled-release systems has proven to have significant advantages comparing with the solvent evaporation technique.

The supercritical antisolvent (SAS) process presented enormous advantages for the preparation of the polymeric microparticles when comparing with conventional technique, as the particles prepared have a smaller size diameter and a narrower particle size distribution.

The process technique used to prepare the delivery system is clearly a factor that influences the percentage of drug impregnated in the polymer microspheres. The particles prepared by solvent evaporation and impregnated using supercritical fluid technology presented a slower release profile and only $40 \%$ of the drug was released after 8-10 h. Microparticles prepared and impregnated using the same technique showed a controlled release profile and almost all the drug was released during what is considered to be the gastrointestinal transit time. These observations are supported by the kinetic constants calculated when Fickian diffusion model was applied to the experimental data. Also, the microspheres prepared in the presence of acetone have a slower release profile and this effect is more pronounced when supercritical fluid technology was used to impregnate the drug in the polymer.

The release profiles of the samples were also evaluated at two different $\mathrm{pHs} 1.2$ and 7.4, simulating respectively the gastric and intestinal environment and it was found that naproxen is preferentially released in a neutral environment.

\section{Acknowledgments}

Ana Rita C. Duarte and Mariana Sousa Costa are grateful for financial support from SFRH/BD/10780/2002 and SFRH/7224/2004/ONMY grants, respectively. This work was financially supported by FCT-MCES, Portugal, under contract POCTI/EQU/46715/2002 from the QCA III/FEDER.

\section{References}

Berens, A.R., Huvard, G.S., Korsmeyer, R.W., Kunig, F.W., 1992. Application of compressed carbon dioxide in the incorporation of additives into polymers. J. Appl. Polym. Sci. 46, 231-242.
Crank, J., 1968. Diffusion in Polymers. Academic Press, London.

Crank, J., 1975. The Mathematics of Diffusion. Oxford Science Publications, Oxford.

Davis, S.S., 1985. The design and evaluation of controlled release systems for the gastrointestinal track. J. Control. Rel. 2, 27-38.

Elvira, C., Fanovich, A., Fernández, M., Fraile, J., Román, J.S., Domingo, C., 2004. Evaluation of drug delivery characteristics of microspheres of PMMA-PCL-Cholesterol obtained by supercritical $\mathrm{CO}_{2}$ impregnation and by dissolution-evaporation techniques. J. Control. Rel. 99, 231240.

Freiberg, S., Zhu, X.X., 2004. Polymer microspheres for controlled drug release Int. J. Pharm. 282, 1-18.

Handbook of Pharmaceutical Excipients, American Pharmaceutical Association, Washington, 4th ed.

Heller, J., 1996. Drug delivery systems, biomaterials science. In: Ratner, B.D., Hoffmann, A.S., Schoen, F.J., Lemons, J.E. (Eds.), An Introduction to Materials in Medicine. Academic Press, London, UK, pp. 346356.

Higuchi, T., 1961. Rate of release of medicaments from ointment bases containing drugs suspension. J. Pharm. Sci. 50, 874-875.

Jung, J., Perrut, M., 2001. Particle design using supercritical fluids: literature and patent survey. J. Supercritical Fluids 20, 179-219.

Kazarian, S.G., 2004. Supercritical fluid impregnation of polymers for drug delivery. In: Supercritical Fluid Technology for Drug Product Development. Marcel Dekker, Inc, pp. 343.

Kikic, I., Sist, P., Applications of supercritical fluids to pharmaceuticals: controlled drug delivery systems, in supercritical fluids: fundamentals and applications, Proceedings of the Second NATO ASI on supercritical fluids, NATO Science series, Kiran, E., Debenedetti, P.G., Peters, C.J. (Eds.), Kluwer Academic Publishers, Dordrecht, The Netherlands, 2000, pp. 291-306.

Kikic, I., Vecchione, F., 2003. Supercritical impregnation of polymers. Curr. Opin. Solid State Mater. Sci. 7, 399-405.

Leo, E., Forni, F., Bernabei, M.T., 2000. Surface drug removal from ibuprofen-loaded PLA microspheres. Int. J. Pharm. 196, 1-9.

Lisa Brannon-Peppas, 1997. Polymers in controlled drug delivery, Med. Plastics Biomater. Available online.

Rekhi, G.S., Jambhekar, S.S., 1995. Ethylcellulose—a polymer review. Drug Dev. Ind. Pharm. 21, 61-77.

Reverchon, E., et al., 2001. Supercritical antisolvent precipitation of salbutamol micro particles. Powder Technology 114, 17-32.

Ritger, P.L., Peppas, N.A., 1987a. A simple equation for description of solute release I. Fickean and non-Fickean release from non-swellable devices in the form of slabs, spheres, cylinders or discs. J. Control. Rel. 5, 23 36 .

Ritger, P.L., Peppas, N.A., 1987b. A simple equation for description of solute release Ii. Fickean and anomalous release from swellable devices. J. Control. Rel. 5, 37-42.

Siempmann, J., Peppas, N.A., 2001. Modeling of drug release from delivery systems based on hydroxypropyl methylcellulose (HPMC). Adv. Drug Del. Rev. 48, 139-157. 